\title{
Molecular dynamics investigation of myelin basic protein stability on lipid membranes
}

\author{
Kyrylo Bessonov, George Harauz* \\ *This study was conducted under the supervision of Professor George Harauz, \\ Department of Molecular and Cellular Biology, \\ University of Guelph, Guelph, Ontario, Canada
}

\begin{abstract}
Simulation of proteins and membranes composed of synthetic lipids on computer clusters provides molecular information that complements experimental data. This paper describes molecular dynamics (MD) approaches to study the properties of biological membranes and proteins using the freely available GROMACS package on the C-terminal $\alpha$-helical peptide of myelin basic protein (MBP). We simulated a mixed membrane - consisting of 2-dimyristoyl-snglycero-3-phosphocholine/1,2-dimyristoyl-sn-glycero-3-phosphoethanolamine (DMPC/DMPE), and a pure DMPC membrane, composed of 188 and 248 lipids, respectively - for $200 \mathrm{~ns}$ at 309K. The DMPC membrane was approximately three times more fluid compared to the DMPC/DMPE system, with the diffusion coefficients (D) being $0.0207 \times 10^{-5} \mathrm{~cm}^{2} / \mathrm{s}$ and $0.0068 \times 10^{-5} \mathrm{~cm}^{2} / \mathrm{s}$, respectively. In addition, we simulated the 14-residue peptide representing the C-terminal $\alpha$-helical region of murine $\mathrm{MBP}$, with sequence $\mathrm{NH}_{2}-\mathrm{A}_{141}$ YDAQGTLSKIFKL ${ }_{154}-\mathrm{COOH}$, in both membrane systems for $200 \mathrm{~ns}$. The negatively-charged $\mathrm{N}$-terminal end of the peptide penetrated further into the DMPC bilayer than into the mixed DMPC/DMPE bilayer. Reduced peptide accessibility to a formal positive charge of the DMPC amine ' $N$ ' atom surrounded by methyl and methylene groups may be the cause [1]. The peptide lost its $\alpha$ helical structure in DMPC/DMPE but not in the DMPC bilayer. These findings show that membrane composition affects MBP's interaction with it, a phenomenon that provides insights into myelin structure - and that may eventually be relevant to understanding the pathogenesis of multiple sclerosis (MS).
\end{abstract}

B iological membranes contain phospholipids that are amphipathic in nature, and thus can effectively interact with a wide variety of molecules. Their main role is to form semi-permeable membranes that act as selective barriers and compartmentalize organelles of the cell. Typical cell membranes have two sheets of lipids that form a hydrated bilayer approximately 4-5 nm thick [2].

Biological membranes play key roles in transport, signal recognition and transduction [3]. Temperature, lipid/protein/cholesterol composition, and bilayer thickness are some of the key parameters that define membrane properties [4]. Modifications in membrane fluidity significantly affect functions such as carriermediated transport, the properties of some membranebound enzymes, phagocytosis, endocytosis, and cell growth [5]. Applications of these discoveries to preliminary gene therapy studies using liposomes are especially promising [6].

In silico simulation of membrane lipids, peptides, and proteins, allows observation of their behavior at the molecular level, and can help one interpret experimental data. This paper describes how to set up and extract useful information from heterogeneous lipid membrane simulations using GROMACS 4.0.5, applied

to mixed DMPC/DMPE and pure DMPC membranes. Although many advanced experimental techniques exist to study membrane properties -- including atomic force microscopy (AFM), X-ray scattering and diffraction, nuclear magnetic resonance (NMR) spectroscopy, and fluorescence recovery after photo-bleaching (FRAP) -in silico molecular dynamics (MD) simulations provide a means to facilitate interpretation of experimental data at an atomic level [3].

\section{BACKGROUND}

\section{Molecular Dynamics (MD): A brief description}

Molecular dynamics investigates the motion of discrete particles under external forces and quantifies them [3]. The interaction of two particles with potential energy produces forces. These forces, with some experimental parameters derived from experimental observations and quantum mechanical calculations, establish the forcefield [7]. The two components of the forcefield comprise interactions between atoms covalently bound, and non-covalent van der Waals and electrostatic interactions between partial charges [3]. 


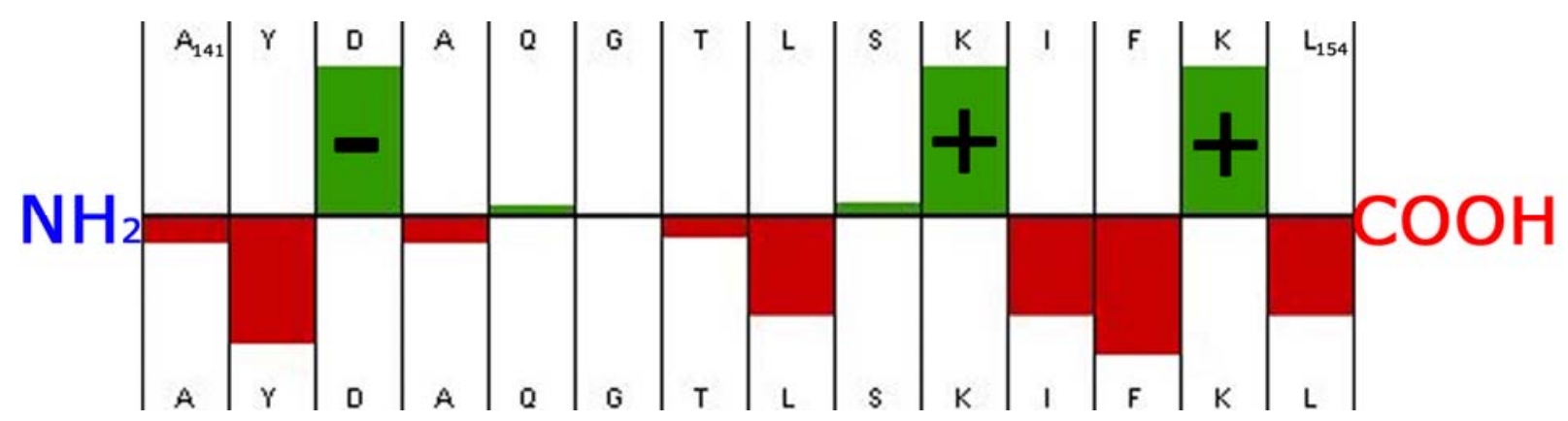

Figure 1: Hydrophilicity plot of the $\mathrm{NH}_{2}-\mathrm{A}_{141}$ YDAQGTLSKIFKL ${ }_{154}-\mathrm{COOH}$ peptide, showing the N- and C-termini. The $\mathrm{Y}$-axis represents the Hopp-Woods hydrophilicity scale, while + and - signs refer to the amino acid R-side chain charge [9].

Simulation of complex systems of hundreds and millions of atoms is achieved by GROMACS (see www.GROMACS.org), one of the fastest and most popular molecular dynamics software packages. GROMACS was designed primarily for simulations of nucleic acids, proteins, and lipids based on non-bonding interactions. The software is not capable of simulations where bonds are broken and reformed, such as chemical / enzymatic reactions. But for situations where nonbonding interactions are expected to predominate, GROMACS can provide information about the behavior of the solute and solvent molecules, and potentially support and explain experimental data.

\section{Myelin basic protein (MBP) - \\ Statement of a biological problem}

MBP is an important protein in the central nervous system, as it facilitates myelin sheath compaction that is responsible for efficient signal propagation [8]. The protein is found in various isoforms with a predominant splice isoform of $18.5 \mathrm{kDa}$ in an adult brain. The main physiological role of MBP is to maintain the myelin sheath that wraps around neurons in the brain and spinal cord, by holding together both cytoplasmic leaflets of oligodendrocyte membranes.

Recent studies have demonstrated how the severity of MS is correlated with post-translational modifications, such as citrullination [10]. Due to its central role, MBP thought to be connected with myelin degradation in the central nervous system in MS. Molecular dynamics provides a powerful means to study the behavior and interaction patterns of MBP with lipid membranes. Such investigations yield information that could provide insights into myelin structure, and eventually into molecular and pathogenic mechanisms in MS. Here, the simulations of DMPC and mixed DMPC/DMPE (with 1:1 molar ratio) membranes, and the MBP C-terminal $\alpha$-helical peptide, were performed on the SHARCNET cluster. The results revealed the important correlation of membrane composition with
MBP behavior, useful to further our knowledge of how myelin is structured.

This article aims to describe both a practical and methodological approach to molecular dynamics through the GROMACS package, as well as to introduce possible applications of such simulations to real biological problems. Thus, it is useful to consult the supplementary material for additional information, key files, and additional programs written in $\mathrm{C}++$ that facilitate the setup of molecular dynamics simulations. (see also: www.uoguelph.ca/ kbessono).

\section{METHODS}

\section{Purpose}

To investigate how both DMPC and DMPC/DMPE membranes affect the behavior of a 14-residue peptide representing one of three $\alpha$-helical regions of the classic murine myelin basic protein (MBP).

\section{The peptide properties}

We modeled the sequence

$$
\mathrm{NH}_{2}-\mathrm{A}_{141} \text { YDAQGTLSKIFKL } 154-\mathrm{COOH}
$$

as a 14-residue long $\alpha$-helical peptide using the program MOE 2008, run under a 32-bit Windows environment. This predicted $\alpha$-helical segment is closest to the C-terminus of the MBP. The simulated peptide segment had an overall +1 charge, and displayed $38 \%$ hydrophilicity based on its primary sequence, as shown in Figure 1. The negativelycharged aspartic acid (D) residue confers -1 charge to the peptide's N-terminus, while two lysine (K) residues ensure $\mathrm{a}+2$ charge at the $\mathrm{C}$-terminal end. The overall peptide $\mathrm{pI}$ is predicted to be 9.6. In silico, the peptide was placed just slightly above the lipid bilayer and simulated for $200 \mathrm{~ns}$.

\section{Results visualization}

For visualization of structural files and trajectories computed by GROMACS, the Visual Molecular 


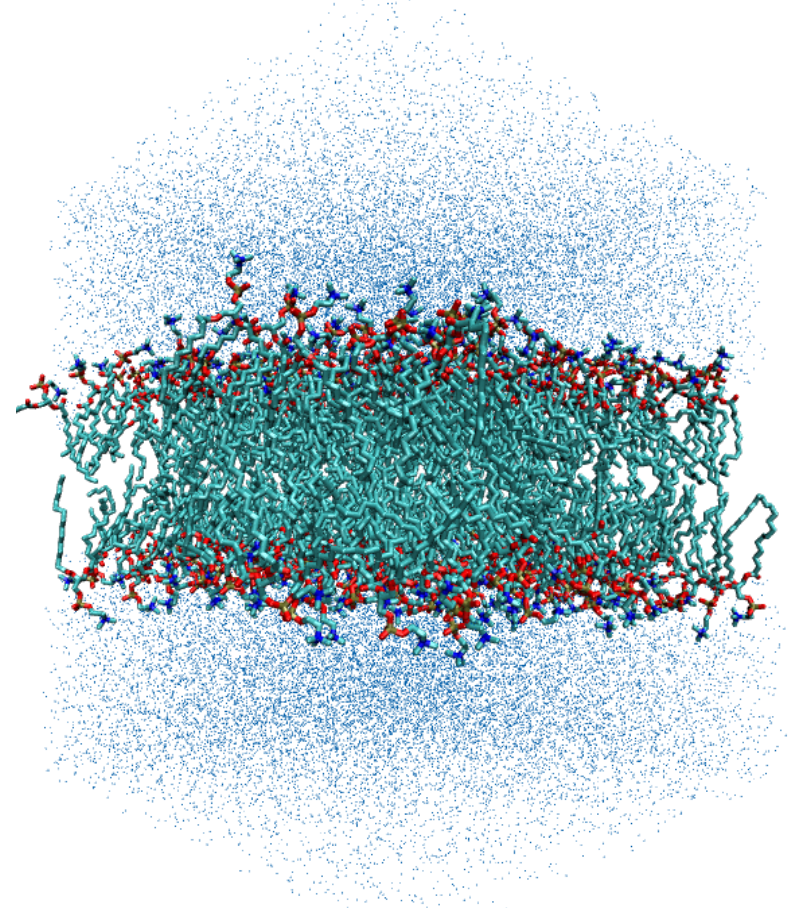

Figure 2: Synthetic phospholipid derivative of phosphocholine (DMPC) bilayer, assembled and

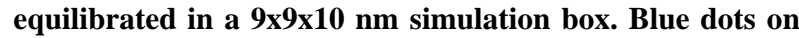
the top and bottom of the DMPC bilayer represent water. DMPC is represented by cyan acyl chains, brown $P$ atoms, and blue $\mathrm{N}$ and red $\mathrm{O}$ atoms.

Dynamics (VMD) program from University of Illinois (www.ks.uiuc.edu/Research/vmd) was extensively utilized. All illustrations were prepared with VMD run on a Linux 32-bit platform.

\section{Getting structural files}

Numerous GROMACS 4.0.5 utilities were employed to generate a suitable simulation system (Figure 2 and 3). The $3 \mathrm{D}$ box system is represented by $9 \times 9 \times 10 \mathrm{~nm}$ virtual space containing all atoms of the simulated system. Our work was based on the publicly accessible DMPC structural coordinates file obtained from Dr. Peter Tieleman's laboratory at the University of Calgary Biocomputing Group with a lipid density of $67 \AA^{2} /$ lipid [11]. A second, mixed DMPC/DMPE membrane was based on work performed by Dr. George Harauz during a visit to Dr. Peter Tieleman's group in early 2009, through a more complicated procedure utilizing in-lab protocols.

\section{Converting structural files to GROMACS file format}

The obtained coordinates files for both membranes were converted to GROMACS file format (*.gro) using the pdb2gmx program. Converting pdb ("protein data bank") format files to GROMACS format (*.gro files) avoids potential compatibility problems and utilizes all features offered by GROMACS. The resultant topology file from pdb2gmx output (topology.top) was essential in describing the composition of the system in terms of molecules, and of their respective dihedral angles and bonding patterns that were modified later. The specified parameter $-b o x$ defined the $\mathrm{x}, \mathrm{y}, \mathrm{z}$ virtual space dimensions of the $3 \mathrm{D}$ simulation box in $\mathrm{nm}$. For example,

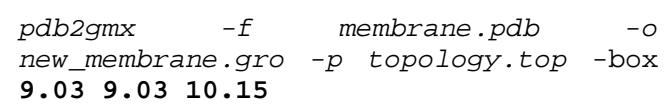

Solvation of the lipid bilayer with water molecules using genbox

Simple point charge water (spc 216) molecules were added to both sides of the lipid bilayers to represent more accurately the hydrated cellular lipid bilayer. Since genbox inserts water molecules randomly, some of them were inserted into the non-polar region of the membrane between acyl chains. Those water molecules were removed by the "water_remover" program, that 
removes any water molecules within defined upper and lower limits along the $\mathrm{x}, \mathrm{y}$ or $\mathrm{z}$ axis of the box (see Supplementary Material). The topology file was updated with the correct number of water molecules calculated with "gro_counter" that specifically counted molecules in gro files (Supplementary material). The lipid bilayer was solvated with spc 216 water molecules as shown below:

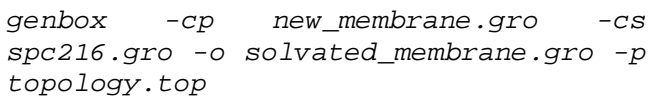

\section{Neutralizing the simulation box}

An overall simulation system zero charge was obtained by adding counter-ions, increasing the stability and reliability of the simulation results. The counter-ions were added to binary tpr files using the genion program. Using the grompp program, it was possible to create a binary tpr file from the simulation files, including an ions.mpd file that defines simulation parameters (Supplementary Material). For example,

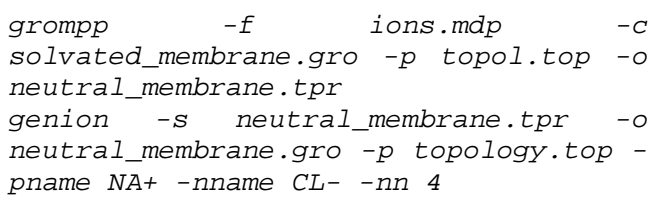

\section{Energy minimization}

To prevent overlap between atoms and increase the stability of the system, energy minimization (EM) using the steepest descents method was first done for both membranes. EM is usually required before any MD run, because lipid membrane solvation in water usually introduces some bad contacts/atom clashes that need to be relaxed before giving kinetic energy (i.e., MD). Execution of parameters in an .mdp file (Supplementary Material) directs mdrun for the optimal positioning of the molecules with the overall lowest possible system energy. Since mdrun requires a binary tpr file, first em.tpr was created with grompp. Parameters describing energy minimization (especially parameter integrator $=$ steep) in the minim.mdp file will run the molecular simulation in energy minimization mode, as in

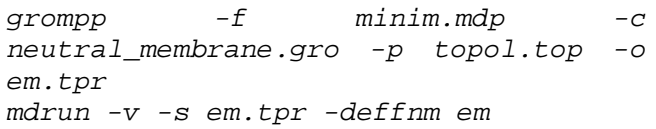

Here, the DMPC and DMPC/DMPE membranes were energy-minimized in approximately 800 steps to 900 $\mathrm{kJ} / \mathrm{mol}$.

\section{Starting the molecular dynamics run}

The molecular dynamics simulations were started on energy-minimized files (em.gro). Since the lipid simulation boxes contained between 30 and 60 thousand molecules, it was necessary to use computer clusters (e.g., SHARCNET). As well, the mdrun program (e.g., mdrun_mpi) was designed to support multithreading of subroutines on multiple processors, taking advantage of the speed of parallel computing. The simulation was run for 200 ns (system trajectory) on 64 CPUs for a total of two weeks (real time). The energy-minimized files were assembled using grompp with simulation parameters described by the md.mdp file. For example,

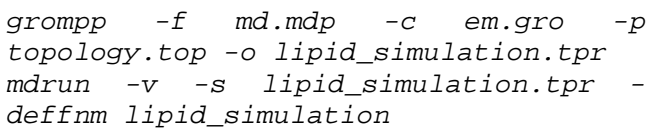

The assembled DMPC and DMPC/DMPE systems were simulated for $200 \mathrm{~ns}$. The obtained trajectory files were analyzed, measuring parameters summarized in Table 1. The g_energy command from the GROMACS package, and the Perl script InflateGro from Dr. Tieleman's research group, were used [11]. Other parameters, such as solvent accessibility, were not successfully measured due to g_sas's difficulty in recognition of the hydrophobic parts of the DMPC molecule.

\section{Visualization and analysis of trajectories}

The 200 ns trajectory files (trr and xtr) were viewed and analyzed with VMD 1.8.6 and GROMACS, comparing parameters such as membrane thickness, membrane area/lipid, and total energies of systems. These simulations could be re-run with different parameters, including different membrane compositions and temperatures or pressures, in order to study their combined or separate effects on the trajectories and overall system stability.

\section{Calculation of membrane thickness}

Membrane thickness was calculated by labeling phosphate atoms of lipid molecules on opposite sides of the bilayer with the help of VMD software. The membrane thickness in DMPC and DMPC/DMPE bilayers did not change significantly during simulation, indicating stability of the membrane. Accurate membrane thickness determination was hindered by constant random lipid motion in both bilayers (data not shown). 


\section{RESUlts AND DisCUSSION}

\section{Measuring membrane properties}

Membrane dynamics simulations provide a means for studying how various conditions such as temperature, protein, cholesterol content, etc., affect membrane characteristics such as fluidity and lipid velocity. The DMPC and DMPC/DMPE bilayers simulation results are summarized in Table 1. All of these parameters are measured by the GROMACS programs shown in Table 1.

The DMPC membrane was found to be more loosely packed than the DMPC/DMPE membrane system, with average areas per lipid of $67 \AA^{2}$ and $55 \AA^{2}$, respectively. It is expected that membranes with a higher density of lipid packing will restrict movement of freely diffusible molecules such as peptides and proteins. Diffusion coefficients (D) describe the mobility of the molecules. Higher D values are indicative of greater mobility. The simulation results in Table 1 indicate that a DMPC bilayer has three times greater fluidity when compared to a mixed DMPC/DMPE bilayer at $309 \mathrm{~K}$. This effect is shown by the diffusion coefficients (D) of $0.0207 \times 10^{-5} \mathrm{~cm}^{2} / \mathrm{s}$ and $0.0068 \times 10^{-5} \mathrm{~cm}^{2} / \mathrm{s}$, respectively. The fluidity difference between the two membranes could be explained partially by the density difference of lipid packing.

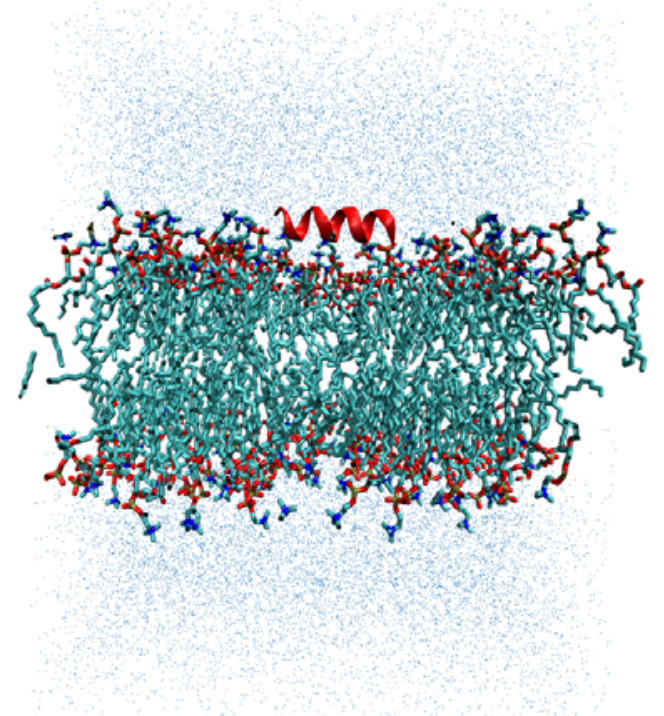

Figure 4: DMPC membrane system with a 14-residues long myelin basic protein (MBP) peptide (red) placed on top of the membrane by merging membrane and peptide structural files using genbox (GROMACS).
Table 1: Summary of measured parameters for DMPC and DMPC/DMPE membrane systems

\begin{tabular}{|l|r|r|}
\hline Membrane & \multicolumn{1}{|c|}{ DMPC } & DMPC/DMPE \\
\hline Total number of atoms & 62613 & 30416 \\
\hline Total number of lipid molecules & 248 & $94 / 94(188)$ \\
\hline Diffusion coefficient (D) $10^{-5} \mathrm{~cm}^{2} / \mathrm{s}$ & 0.0207 & 0.0068 \\
\hline Kinetic Energy (J/mol) & 161870 & 78602 \\
\hline Total Energy (J/mol) & -891474 & -477439 \\
\hline Heat Capacity $\mathrm{C}_{\mathrm{v}}\left(\mathrm{J} / \mathrm{mol}^{\star} \mathrm{K}\right)$ & 12.4721 & 12.4724 \\
\hline Temperature $(\mathrm{K})$ & 309 & 309 \\
\hline Pressure (bar) & 1.66 & 1.097 \\
\hline Average Area per lipid $(\AA 2 /$ lipid) & 67.26 & 55.24 \\
\hline Membrane Thickness $(\mathrm{A})$ & $33.9-35.72$ & $35.8-38$ \\
\hline
\end{tabular}

Simulation of 14-residue long MBP C-terminal peptide

In addition to pure membrane simulations, adding a 14residue long myelin basic protein peptide to each bilayer system showed differences in protein stability and depth of penetration into the membrane over the course of the simulation. Figures 4 to 7 show the initial and intermediate stages of the simulation. Overall, the peptide penetrated further into the DMPC than into the mixed DMPC/DMPE membrane. Helical secondary structure retention was stronger in the DMPC bilayer system.

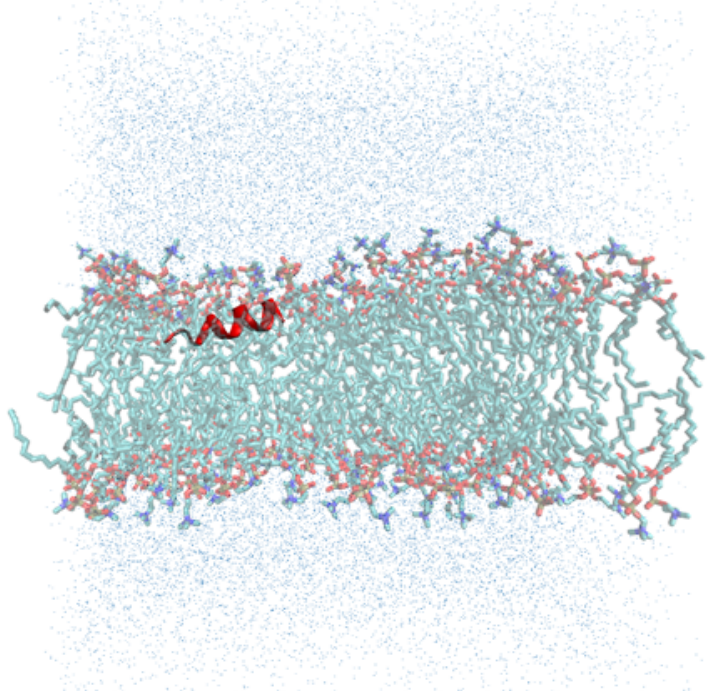

Figure 5: DMPC lipid bilayer with a 14-residue MBP peptide after an 80 ns simulation. Note that the $\mathrm{N}$ terminus of the peptide has deeply penetrated at least halfway into the leaflet. The helical structure of the peptide is preserved, as seen by comparison with the initial state (Figure 3). 


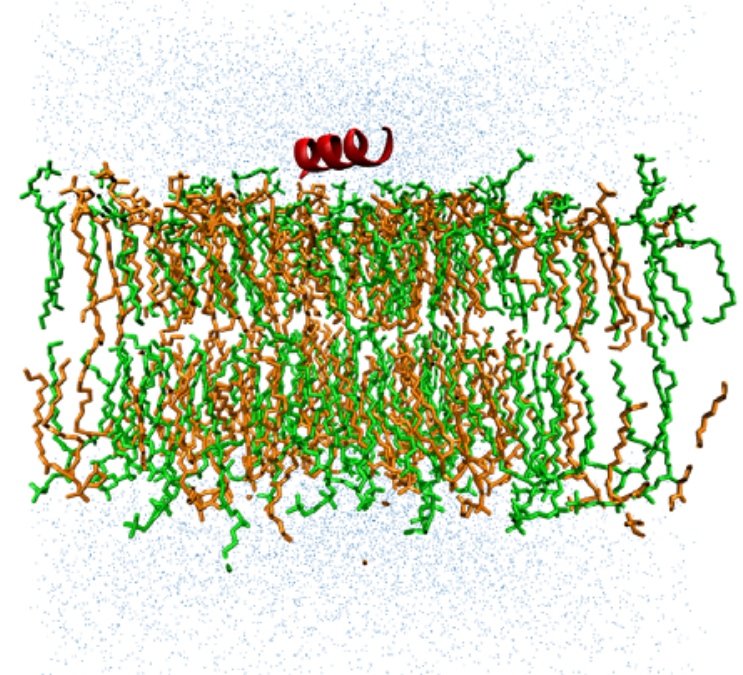

Figure 6: DMPC/DMPE mixed membrane with the same 14-residue long myelin basic peptide (red) positioned on top of the membrane. This snapshot represents the initial position of the peptide.

\section{Membrane stability differences by MBP C-terminal peptide}

The peptide in the DMPC bilayer showed greater secondary structure stability compared to the mixed DMPC/DMPE bilayer. We hypothesize that DMPE molecules may play a role in destabilization of the peptide $\alpha$-helix through electrostatic interactions preventing its entry into the lipid bilayer as explained below. This finding suggests that localized denaturation of solvent accessible MBP $\alpha$-helices could occur within myelin, possibly augmenting the myelin degradation in MS, resulting eventually in subsequent decrease of nerve impulse propagation efficiency.

\section{Membrane penetration differences by MBP C- terminal peptide}

We also assessed the penetration depth of the same 14residue long peptide. The DMPC bilayer was penetrated more strongly by the peptide than the DMPC/DMPE bilayer, further highlighting the importance of the membrane compositions (Figures 5 and 7). Synthetic DMPC and DMPE molecules represented phosphocholine (PC) and phosphoethanolamine (PE). Substitution of ethanolamine for choline in the bacterial cell wall significantly alters important biological functions, such as cellular adhesion and bacterial transformation [12]. As seen in Figure 8, choline has

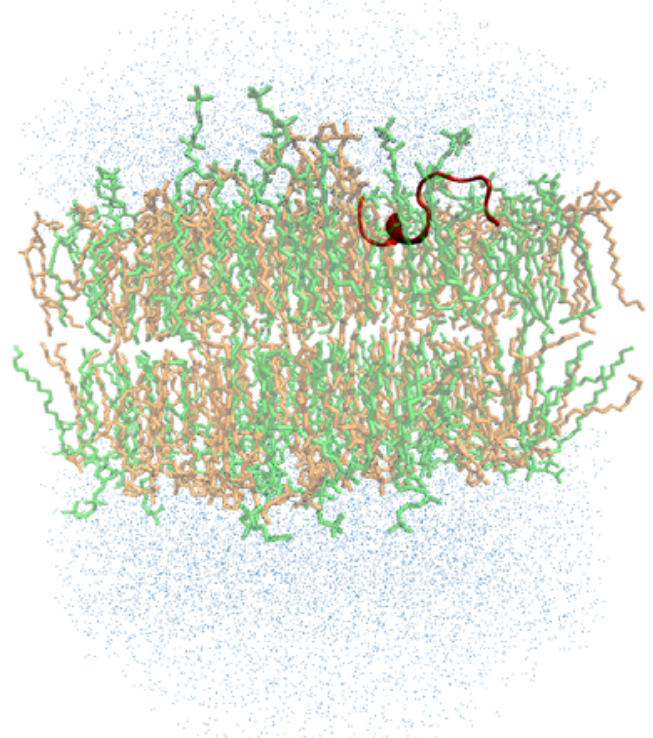

Figure 7: DMPC/DMPE mixed bilayer with 14-residue peptide of myelin basic protein after an 80 ns simulation. Observe that the helical structure has been greatly unwound. The peptide penetrated the DMPC/DMPE bilayer less significantly compared to the DMPC bilayer, due to electrostatic interactions and a decrease in steric hindrance (compare to Figure 4).

three methyl $\left(\mathrm{CH}_{3}\right)$ groups attached to an ' $\mathrm{N}$ ' atom, whereas ethanolamine has three $\mathrm{H}$ atoms instead. Both choline and ethanolamine have the same formal charge of +1 , but behave differently [1]. Here, the peptide remained attached to the DMPC/DMPE membrane surface, not being able to penetrate further into it. The opposite was observed in simulations with a DMPC bilayer into which the peptide penetrated deeply. Zull and Hopfinger [1] measured the accessibility of a negative test charge to a positively-charged amide ' $\mathrm{N}$ ' atom, concluding that ethanolamine interacts more strongly with anions. Even though choline lipids have a three times stronger partial positive charge on an ' $\mathrm{N}$ ' atom compared to ethanolamine $(+0.685$ against +0.131 ), it is sterically poorly accessible, resulting in choline having poorer interactions with anions. The positive charge of ethanolamine on an ' $\mathrm{N}$ ' atom is more diffused and more accessible [1], possibly resulting in stronger interactions with the negative N-terminus of the peptide. Here, the peptide interacted more strongly with DMPE lipids in the mixed DMPC/DMPE membrane compared to the pure DMPC membrane.

\section{CONCLUSIONS}

We have presented the results of MD simulations of DMPC and DMPC/DMPE bilayers, both alone and with 


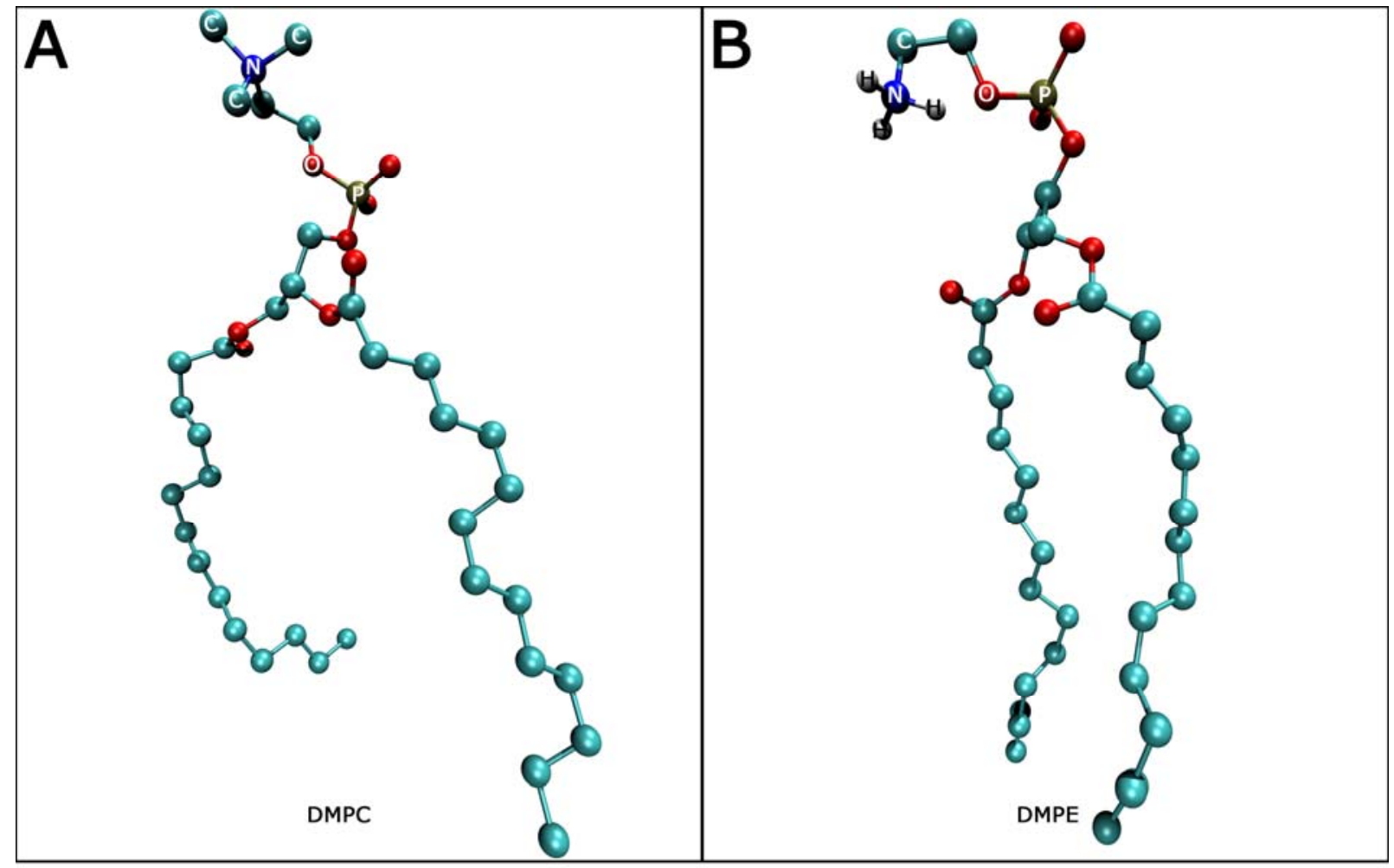

Figure 8: (A) DMPC to (B) DMPE lipid structural comparison, illustrating differences in accessibility to formal positive charge of the $\mathrm{N}$ atom. Some important atoms around the $\mathrm{N}$ atom are labeled with white letters. Carbon atoms connected to ' $N$ ' in DMPC represent $\mathrm{CH}_{3}$ groups.

a peptide fragment of MBP. We were successful in measuring key parameters such as diffusion coefficients and membrane thickness. In addition, we have described a general method for performing the workflow required to set up the molecular simulation of lipid membranes. The DMPC membrane showed a greater degree of fluidity at $309 \mathrm{~K}$ compared to the mixed DMPC/DMPE membrane, with diffusion coefficients of $0.0207 \times 10^{-5} \mathrm{~cm}^{2} / \mathrm{s}$ and $0.0068 \times 10^{-5}$ $\mathrm{cm}^{2} / \mathrm{s}$, respectively. Also the DMPC membrane was more deeply penetrated by a 14-residue long $\alpha$-helical peptide $\left(\mathrm{A}_{141}\right.$ YDAQGTLSKIFKL $\left._{154}\right)$ than the mixed DMPC/DMPE bilayer.

The results show the usefulness of molecular dynamics approaches in studying molecular dynamics of biological membranes. Although requiring care to set up, this approach is useful to study various factors affecting biological membranes. Our preliminary results suggest that local changes in membrane composition (e.g., enrichment in DMPE molecules), as well as the electrostatic properties could ultimately cause localized denaturation and mobility changes of external MBP $\alpha$ helices. We suggest that localized denaturation of solvent accessible MBP $\alpha$-helices could possibly augment the degradation of myelin in MS, resulting eventually in subsequent decrease of nerve impulse propagation efficiency. Thus, membrane microdomain lipid composition could affect the local degree of compaction of myelin.

The above results highlight the importance of the membrane composition, in conjunction with an array of other membrane properties, on final protein structural stability and behavior that is ultimately reflected in its biological function. These findings further support evidence from other studies that protein-membrane interactions and $\alpha$-helical protein stability are governed by a combination of factors including: hydrogen bonds, ion pairs, favorable surface van der Waals interactions, and thermodynamic parameters [13].

\section{REFERENCES}

1. Zull, J.E., Hopfinger, A.J. 1969. Potential energy fields about nitrogen in choline and ethanolamine: biological function at cellular surfaces. Science 165, 512-513.

2. Kuchel, P.W., Ralston, G.B. 1988. Theory and Problems of Biochemistry. New York: Schaum's Outline Series/McGraw-Hill

3. Kandt, C., Ash, W.L., Tieleman, D.P. 2007. Setting up and running molecular dynamics simulations of membrane proteins. Methods 41, 475-488.

4. Martin, C.E., Hiramitsu, K., Kitajima, Y., Nozawa, Y., Skriver, L., Thompson, G. A., 1976. Molecular 
control of membrane properties during temperature acclimation. Fatty acid desaturase regulation of membrane fluidity in acclimating Tetrahymena cells. Biochemistry 15, 5218-5227.

5. Spector, A.A., Yorek, M.A. 1985. Membrane lipid composition and cellular function. J Lipid Res 26, 1015-1035.

6. Jeschke, M. G., Klein, D. 2004. Liposomal gene transfer of multiple genes is more effective than gene transfer of a single gene. Gene Ther 11, 847855.

7. Ponder, J.W., and Case, D.A. 2003. Force fields for protein simulations. Adv. Prot. Chem. 66: 27-85.

8. Harauz, G., Ladizhansky, V., Boggs, J. M. 2009. Structural polymorphism and multifunctionality of myelin basic protein. Biochemistry 48, 8094-8104.
9. Hopp T.P., Woods K.R. 1981 Prediction of protein antigenic determinants from amino acid sequences Proc.Natl. Acad. Sci. 78, 3824-3828.

10. Harauz, G., Musse, A. A. 2007. A tale of two citrullines--structural and functional aspects of myelin basic protein deimination in health and disease. Neurochem Res 32, 137-158.

11. Tieleman, D.P. 2009. Structures and Topologies. Available online from: http://moose.bio.ucalgary.ca/index.php?page=Struc tures_and_Topologies Accessed 21 April 2010.

12. Tomasz, A. 1967. Choline in the cell wall of a bacterium: novel type of polymer-linked choline in Pneumococcus. Science 157, 694-697.

13. Popot, J.L., Engelman, D.M. 2000. Helical membrane protein folding, stability, and evolution Annu Rev Biochem 69, 881-922. 